\title{
IATF DE FÊMEAS BOVINAS EM DIFERENTES PERIODOS PÓS-PARTO
}

\author{
Mariana Antônia Pedroso ${ }^{1}$, Adalgiza Pinto Neto ${ }^{1}$, Eduardo Custódio dos Santos ${ }^{2}$, \\ Jonatas Cattelam ${ }^{1}$, Marcelo Falci Mota ${ }^{1}$ \\ 1Universidade Federal da Fronteira Sul - UFFS, Campus Realeza, Realeza, PR, Brasil \\ ${ }^{2}$ Médico Veterinário Autônomo, Santa Izabel do Oeste, PR, Brasil \\ ${ }^{3}$ Universidade Estadual de Maringá - UEM, Campus Umuarama, Umuarama, PR, Brasil \\ E-mail: adalgiza.uffs@gmail.com; adalgiza.neto@uffs.edu.br
}

\section{Recebido em: 22/09/2018 - Aprovado em: 23/11/2018 - Publicado em: 03/12/2018 DOI: 10.18677/EnciBio_2018B53}

Em bovinos, a utilização da Inseminação Artificial em Tempo Fixo (IATF) serve como ferramenta para aumentar os índices reprodutivos e a rentabilidade. Objetivou-se avaliar a taxa de gestação de fêmeas bovinas em diferentes períodos pós-parto, submetidas à IATF. Para tanto, 77 fêmeas (oito novilhas, 22 primíparas e 47 vacas), da Raça Nelore, em diferentes períodos pós-parto, foram submetidas a exame ginecológico completo, avaliação da presença de corpo lúteo ovariano $(C L)$ e escore de condição corporal (ECC - escala de zero a cinco). Na ausência de alterações reprodutivas foram submetidas ao seguinte protocolo hormonal: dia zero-manhã, aplicação intramuscular (IM) de $2 \mathrm{mg}$ de benzoato de estradiol (Sincrodiol囚) e implantação de dispositivo intravaginal de progesterona de primeiro uso (Sincrogest®); dia nove-manhã, retirada do dispositivo de progesterona, aplicação IM de 0,52mg de PGF2alfa (Sincrocioß) e de 300UI de eCG (Sincro eCG®); dia 10tarde aplicação IM, 0,01mg de GnRH (Sincro forte®) e no dia 11-manhã, a IATF utilizando-se sêmen de touros de diferentes raças e qualidade comprovada. As fêmeas não gestantes, diagnosticadas pela ultrassonografia, no dia 56 foram novamente submetidas ao protocolo anterior, porém utilizando-se implante de progesterona de segundo uso. Nas condições desse estudo, concluiu-se que a taxa de gestação foi semelhante entre as fêmeas, independente da categoria, do período pós-parto, da presença de CL no dia zero, da reutilização do dispositivo de progesterona e da raça do touro. No entanto, fêmeas com maior ECC apresentaram taxa de gestação superior.

PALAVRAS-CHAVE: IATF, sincronização de estro, taxa de gestação.

\section{IATF IN BOVINE FEMALES ON DIFFERENT POST-BREASTFEED PERIODS}

\begin{abstract}
In cattle, the use of Artificial Insemination in Fixed Time (AIFT) serves as a tool to increase reproductive rates and profitability. The aim of this study was to evaluate the gestation rate of bovine females in different postpartum periods, submitted AIFT. Therefore, 77 females (eight heifers, 22 primiparous and 47 cows), of the Nelore Breed, in different postpartum periods, were submitted to a complete gynecological examination, evaluation of the presence of ovarian corpus luteum (CL) and body condition score (BCS - scale from zero to five). In the absence of reproductive
\end{abstract}


changes were submitted to the following hormonal protocol: day zero-morning, intramuscular application (IM) of $2 \mathrm{mg}$ Estradiol Benzoate (Sincrodiol ${ }^{\circledR}$ ) and implantation of intravaginal progesterone device of first use (Sincrogest ${ }^{\circledR}$ ); day nine morning, withdrawal of the progesterone device, application IM of 0,52mg PGF2 $\alpha$ (Sincrocio ${ }^{\circledR}$ ) and $300 \mathrm{UI}$ of eCG (Sincro eCG®); day 10-in the afternoon application IM $0,01 \mathrm{mg}$ of $\mathrm{GnRH}$ GnRH (Sincro forte®) it's at 11-mornig, at AIFT using semen of bulls of different races and proven quality. Non-pregnant females, diagnosed by ultrasonography, on day 56 were again submitted to the previous protocol, but using a second-use progesterone implant. Under the conditions of this study, it was accomplish that the gestation rate was similar among females, independent of the category, of the postpartum period, the presence of CL on day zero, reuse of the progesterone device and bull breed. However, females with greater BCS presented higher gestation rate.

KEYWORDS: AIFT, synchronize estrus, pregnancy rate.

\section{INTRODUÇÃO}

A pecuária brasileira enfrenta desafios relacionados à produtividade e a concorrência com as demais fontes de origem animal (CABRAL et al., 2013). Os índices produtivos e reprodutivos do rebanho estão sujeitos aos efeitos diretos e indiretos do clima, relacionados ao sistema extensivo de criação predominante, influenciados ainda pela raça, particularidades individuais, escore de condição corporal (ECC) e nutrição (CABRAL et al., 2013; CUNHA et al., 2013).

Sabendo-se disso, inúmeras biotécnicas reprodutivas vem sendo continuamente desenvolvidas afim de beneficiar a atividade, como a IATF, que visa predeterminar um momento para realização de inseminação das fêmeas afim de facilitar as atividades, reduzindo a mão de obra necessária e concentrando os manejos envolvidos (CUNHA et al., 2013). A técnica baseia-se em manejar as fêmeas com aplicação de hormonios para manipulação e sincronizaçãdo do ciclo estral e ovulação (SILVA; PIMENTEL, 2017).

Estas biotecnologias voltadas à reprodução têm melhorado a produtividade e a genética do rebanho bovino nacional, aumentando a eficiência e desenvolvendo gradativamente a cadeia pecuária (ZANGIROLAMO et al., 2017).

Os tratamentos hormonais visando IATF têm demonstrado eficácia justamente pela ausência da detecção de estro, refletindo em maior praticidade, aumento na taxa de gestação e diminuição do anestro pós-parto (BÓ; BARUSELLI, 2014). Além disso, o dispositivo intravaginal pode servir como suplemento hormonal para animais com diminuição da fertilidade (COLAZO; MAPLETOFT, 2014).

Atualmente, há inúmeras possibilidades relativas ao protocolo de IATF, pois variações fisiológicas, de raças, de condição reprodutiva e/ou nutricional, influenciam significativamente os resultados, devendo sempre serem consideradas para escolha do melhor protocolo para cada situação (TORRES-JÚNIOR et al., 2016).

A inseminação artificial (IA) é utilizada visando o melhoramento genético e reprodutivo de rebanhos. Porém a detecção de estro se faz necessária, tornando-se um fator limitante, o que a difere da IATF, que considera o momento das aplicações hormonais para realização da IA, sem a necessidade de percepção de estro (SILVA; PIMENTEL, 2017; ZANGIROLAMO et al., 2017). Assim, a IATF surge como uma alternativa amplamente difundida, que visa aumentar o número de fêmeas inseminadas através da aplicação de protocolos práticos e de fácil execução, evitando perdas econômicas e diminuindo o intervalo de partos (BÓ et al., 2013; BÓ; BARUSELLI, 2014; SILVA; PIMENTEL, 2017). 
Porém, os fármacos a serem utilizados para promover tais eventos fisiológicos em programas de IATF devem ter uma relação custo/benefício rentável para o produtor (ALMEIDA et al., 2006) considerando inclusive, que o acesso aos pequenos produtores ainda é limitado (SILVA; PIMENTEL, 2017). Entre os hormônios utilizados para desenvolver os protocolos, o dispositivo a base de progesterona é o que apresenta maior custo, muitas vezes inviabilizando sua utilização, o que justifica a busca por alternativas capazes de ajustar a relação custo/benefício dos protocolos, por meio da reutilização desse dispositivo (PINTONETO et al., 2009).

A partir da possibilidade de reutilização do dispositivo de progesterona, os custos da implantação dos programas de melhoramento genético com IATF tendem a ser menos onerosos (FARIAS et al., 2015).

Dessa forma, objetivou-se com esse estudo avaliar a taxa de gestação de fêmeas bovinas, em diferentes períodos pós-parto, submetidas à sincronização e ressincronização de estro visando IATF.

\section{MATERIAL E MÉTODOS}

O protocolo experimental desse estudo foi submetido e aprovado pela Comissão De Ética No Uso De Animais (CEUA) da Universidade Federal da Fronteira Sul-UFFS (Protocolo23205.000710/2017-95).

Após aprovação do CEUA, o estudo foi desenvolvido no período de maio a agosto de 2017, em uma propriedade rural localizada no Município de Planalto, na Região Sudoeste do Paraná, com altitude de 376 metros, latitude 25 $42^{\prime}$ 58'S, longitude equivalente a 5345' 58'’W e clima subtropical úmido.

Foram utilizadas 77 fêmeas bovinas da raça Nelore, sendo oito novilhas, 22 primíparas e 47 vacas em diferentes períodos pós-parto, com ou sem bezerro ao pé, mantidas sob pastejo de Brachiaria spp, suplementadas com sal mineral proteinado e água ad libitum.

Todas as fêmeas foram submetidas a exame ginecológico completo, avaliação da presença de corpo lúteo ovariano, e na ausência de alterações reprodutivas foram submetidas a um protocolo de sincronização de estro e ovulação, seguido pela IATF. Para tanto, no dia zero (D0) pela manhã, avaliou-se o escore da condição corporal (ECC) em escala de zero a cinco (FERREIRA et al., 2013), aplicou-se $2 \mathrm{mg}$ de benzoato de estradiol IM (Sincrodiol囚) e implantou-se um dispositivo intravaginal de progesterona de primeiro (Sincrogest®). No dia nove (D9) pela manhã, retirou-se o dispositivo de progesterona, aplicou-se via IM 0,52mg de PGF2alfa (Sincrocio®) e 300UI de eCG (Sincro eCG®). No dia 10 (D10) a tarde aplicou-se por via IM 0,01mg de $\mathrm{GnRH}$ (Sincro forte®) e no dia 11 (D11) pela manhã, realizou-se a IATF, utilizando-se sêmen de qualidade comprovada, oriundos de touros de três raças.

Aos 45 dias após a IATF, realizou-se o diagnóstico de gestação, através de ultrassonografia (SUIU® CTS800) (FREITAS et al., 2007). As fêmeas diagnosticadas não gestantes, foram novamente submetidas a exame ginecológico, e na ausência de alterações reprodutivas, realizou-se o mesmo protocolo anterior, no entanto, utilizando-se dispositivo de progesterona de segundo uso.

Para análise dos dados, utilizou-se o Teste do Qui-quadrado para avaliação da taxa de gestação considerando categoria da fêmea (novilha, primípara ou vaca), período pós-parto (zero, até 120, 120-210, 210-310 ou acima de 310 dias), presença de corpo lúteo ovariano no Do (com ou sem corpo lúteo), uso do dispositivo intravaginal de progesterona (novo ou reutilizado), raça do touro (Angus, Braford ou 
Gir) e ECC (inferior a três, de 3-3,5 ou superior a quatro pontos), utilizando-se o SAS (Versão 9.4), com 5\% de significância.

\section{RESULTADOS E DISCUSSÃO}

Novilhas, primíparas e vacas apresentaram taxa de gestação semelhante (p>0,05), sendo de 23,08\% (3/13), 51,61\% (16/31) e de 37,84\% (28/74), respectivamente (Figura 1-B). Esses dados foram divergentes daqueles relatados por Almeida et al. (2006), onde primíparas obtiveram taxa de gestação menor ao realizar IATF quando comparada a multíparas, $35 \%$ e $52,7 \%$ de gestação respectivamente. Medalha et al. (2015), ao sincronizarem 116 primíparas e 1060 multíparas, concluíram que vacas primíparas apresentaram menor taxa de gestação em relação às pluríparas (34,48 \% - 40/116 e 50,85 \% - 539/1060, respectivamente).
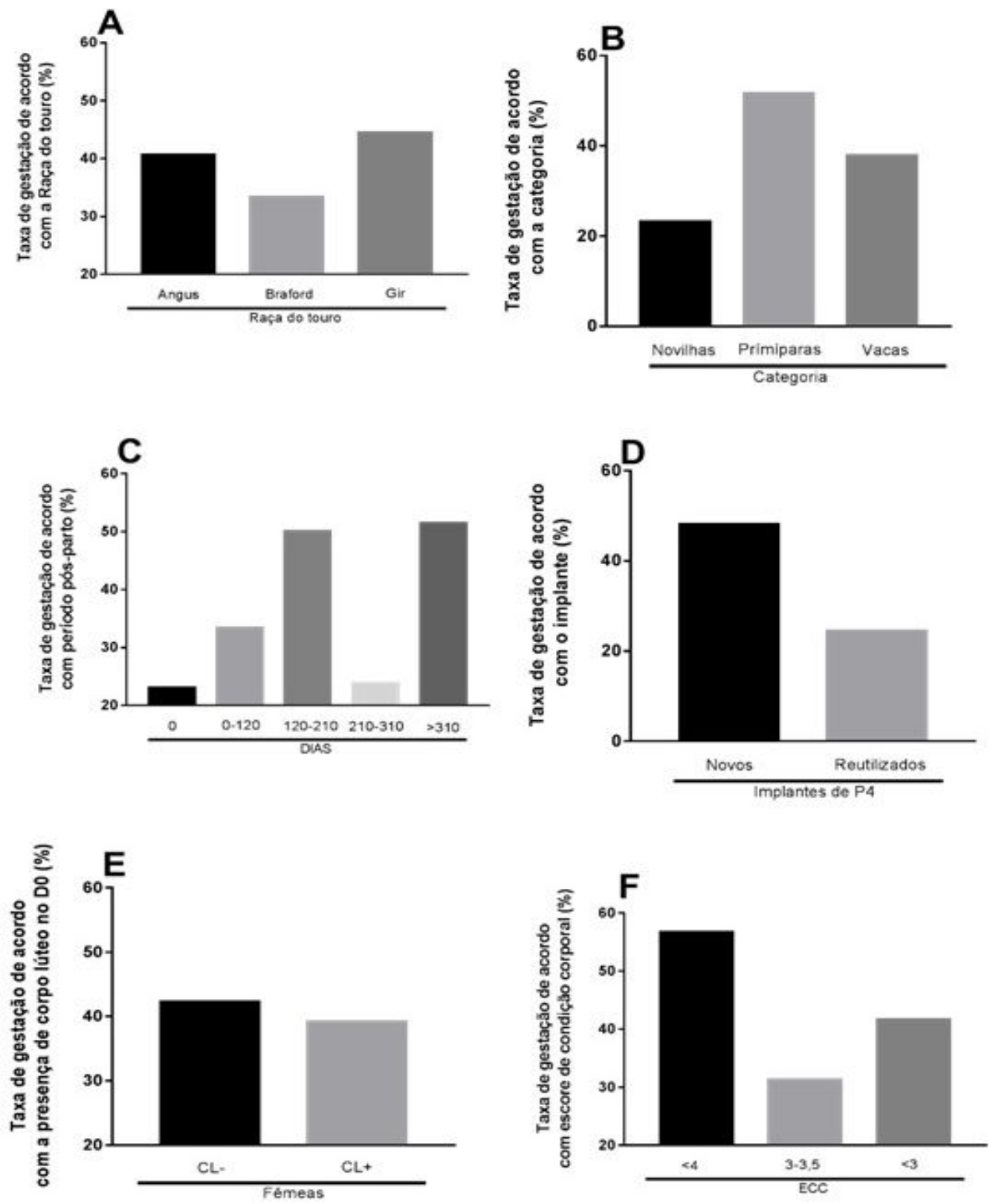

FIGURA 1. Taxa de gestação em fêmeas bovinas submetidas a IATF, ao se considerar a raça do touro (A), a categoria das fêmeas $(B)$, período pós-parto $(C)$, reutilização do dispositivo de progesterona (D), presença de CL no D0 (E) e o ECC (F). Fonte: autores.

Nesse estudo, ao se agrupar os animais de acordo com o período pós-parto, ENCICLOPÉDIA BIOSFERA, Centro Científico Conhecer - Goiânia, v.15 n.28; p.622 
em zero (novilhas), até 120 dias, 120-210 dias, 210-310 dias e acima de 310 dias pós-parto, não se observou diferença na taxa de gestação após a IATF, sendo de $23,08 \%$ (3/13), 33,33\% (7/21), 50,00\% (14/28), 23,81\% (5/21) e 51,43\% (18/35), respectivamente $(p>0,05)$ (Figura 1-C). De forma semelhante, Gottschall et al. (2009) não encontraram diferença na taxa de gestação em dois grupos experimentais com diferentes períodos pós-parto, sendo de 89,1 e 65,1 dias pós-parto.

Adicionalmente, Meneghetti e Vasconcelos (2008) não verificaram efeitos da condição corporal e do período pós-parto na taxa de concepção nos animais sincronizados para IATF. Ainda, Gunn et al. (2015) compararam a taxa de gestação em 879 matrizes sincronizadas para IATF, agrupadas em primíparas e multíparas, e em diferentes períodos pós-parto, e concluíram que não houve efeito do pós-parto na taxa de gestação.

Fêmeas que apresentaram corpo lúteo no D0, tiveram taxa de gestação semelhante aquelas sem corpo lúteo, sendo de 42,31\% (11/26) e de 39,13\% (36/92), respectivamente ( $p>0,05)$ (Figura 1-E). Barbosa et al. (2011), observaram que a presença ou não do corpo lúteo no dia zero do protocolo não afetou os resultados de taxas de ovulação ou de concepção das vacas ovuladas. Rodrigues et al. (2015) também constatou que a presença de corpo lúteo não afetou a performance reprodutiva de fêmeas Nelore submetidas ao protocolo de IATF.

De forma semelhante, a reutilização de dispositivo de progesterona também não interferiu na taxa de gestação $(p>0,05)$, sendo que fêmeas que receberam dispositivos novos tiveram $48,05 \%$ (37/77) e aquelas que receberam reutilizados, 24,39\% (10/41) de taxa de gestação (Figura 1-D). De acordo com Medalha et al. (2014), o uso de dispositivos intravaginais de progesterona novos ou reutilizados, durante oito ou 16 dias, não levaram a diferenças na taxa de gestação, viabilizando a reutilização por até três vezes, quando utilizado juntamente com o cipionato de estradiol e eCG em protocolos de IATF.

A fim de avaliar a influência do número de utilização do dispositivo de progesterona na taxa de gestação, Silva et al. (2017) analisaram os dados de concepção de 1791 fêmeas zebuínas paridas, submetidas à IATF com dispositivo de diferentes usos, e demonstraram que o dispositivo Sincrogest ${ }^{\circledR}$ pode ser utilizado até três vezes no protocolo de IATF de vacas zebuínas paridas, com resultados satisfatórios.

Farias et al. (2015) utilizaram como modelo experimental 299 vacas multíparas, e não foram observadas diferenças nas taxas de gestação em relação à quantidade de uso do dispositivo intravaginal de progesterona, sendo que dispositivos de primeiro uso foram efetivos em $76 \%$ (114/150) dos casos, muito semelhante aos de segundo uso, que apresentaram efetividade de $75,87 \%$ (113/149), exercendo um feedback negativo sobre o eixo hipotálamo-hipófise, permitindo reduzir as concentrações circulantes deste hormônio no momento da sua retirada, aumentando a pulsatilidade do LH para aumentar o diâmetro folicular e ovulação, com aumento da taxas de concepção e formação de corpo lúteo funcional.

Medalha et al. (2015) também avaliaram o efeito do número de utilizações dos dispositivos intravaginais de progesterona, obtendo resultados de 44,8\% (156/348), $51,1 \%(213 / 417)$ e $51,1 \%(210 / 411)$, para os grupos de primeiro, segundo e terceiro uso respectivamente. Reforçando a eficiência destes dispositivos quando reutilizados.

Ao sincronizar o estro visando a IATF, com implantes auriculares impregnado com progesterona (Crestar $\AA$ ), em 241 vacas nelore puras de origem, verificou-se 
taxa de gestação de 48,3\% e 48,7\%, ao utilizarem implantes novos e reutilizados, respectivamente. Assim como na IATF, quando avaliada a taxa de gestação em estação de monta natural, a reutilização de implantes mostrou-se viável, com taxas de $85,2 \%$ para implantes novos e $86,5 \%$ para implantes reutilizados (ALMEIDA et al., 2006).

A raça do touro não interferiu na taxa de gestação ( $p>0,05)$, sendo de 40,63\% (26/64), 33,33\% (9/27) e 44,44\% (12/27), para as raças Angus, Braford e Gir, respectivamente (Figura 1-A). Ereno et al. (2007) relataram que ao sincronizar 230 vacas Nelores e mestiças (Nelore x Red Angus) para a IATF, utilizando-se sêmen de três diferentes touros da raça Nelore não demonstrou interferência nos resultados encontrados. Medalha et al. (2015), avaliaram 1176 vacas zebuínas submetidas a IATF em diferentes propriedades de dois Estados, e concluiu que o touro não influenciou a taxa de gestação, concordando com os resultados obtidos neste trabalho.

Nesse estudo, ao se analisar a condição corporal, observou-se que fêmeas com ECC acima de quatro pontos, apresentaram taxa de gestação superior $(p<0,05)$ $(56,67 \%$ - 17/30) aquelas com ECC $3-3,5$ pontos $(31,25 \%$ - 20/64) e semelhante a taxa de gestação das fêmeas com ECC inferior a três $(41,67 \%-10 / 24)(p>0,05)$. No entanto, fêmeas com ECC inferior a três e fêmeas entre 3-3,5, apresentaram taxa de gestação semelhantes ( $p>0,05)$ (Figura 1-F).

Correa-Orozco et al. (2013) conduziram experimento para avaliar os fatores que afetam a gestação em vacas Brahman submetidas à IATF, e concluiram que a condição corporal não afetou a taxa de gestação. De mesmo modo, Cunha et al. (2013), utilizaram 350 vacas com média de ECC 2,65 \pm 0,01, primíparas lactantes, e não observaram qualquer diferença relacionada ao número de dias pós-parto e ECC entre os grupos de fêmeas, contrariando os resultados obtidos neste estudo.

Ao utilizar 181 vacas multíparas da raça Nelore, com mais de cem dias pósparto, mantidas sob pastejo e distribuídas em dois grupos de acordo com o ECC (escala de 1-5) e submetidas ao mesmo protocolo de sincronização, Ferreira et al. (2013), verificaram que 96 vacas com ECC $\geq 3,0$ e 85 vacas com ECC $\leq 2,5$, apresentaram taxa de gestação de $86,5 \%$ e $65,9 \%$, respectivamente, sugerindo que a condição corporal pode afetar o desempenho reprodutivo de vacas Nelore, observando-se maior desempenho reprodutivo em fêmeas com melhor condição corporal, concordando com o que foi observado nesse estudo.

Meneghetti e Vasconcelos (2008), ao avaliarem o efeito da condição corporal e dos dias pós-parto na resposta ao protocolo de IATF em 538 vacas, sobre a taxa de sincronização, relataram que o aumento na taxa de gestação foi proporcional ao aumento na condição corporal dos animais estudados, de forma que o incremento médio na concepção foi de seis pontos percentuais para cada 0,25 ponto na condição corporal, e animais com menor condição corporal tiveram menor taxa de sincronização.

Ao avaliar 222 vacas de corte com cria ao pé, de 3 a 9 anos de idade, da raça Montana, foi observado que o ECC influenciou as taxas de gestação à IATF, com associação positiva entre o aumento do ECC e a gestação, apresentando diferença na resposta em animais com ECC superior ou inferior a 2,5. O ECC influenciou também na taxa de manifestação de estro. Vacas com ECC $\geq 2,5$ apresentaram maior taxa de manifestação de estro do que vacas com ECC < 2,5 (GOTTSCHALL et al., 2012).

\section{CONCLUSÃO}

Nas condições desse estudo, concluiu-se que a raça do touro, a categoria das 
fêmeas (novilhas, primíparas ou multíparas), o período pós-parto e a reutilização de dispositivo de progesterona não interferiram na taxa de gestação após IATF dos animais estudados. No entanto, a taxa de gestação foi influenciada pelo ECC das fêmeas estudadas.

\section{REFERÊNCIAS}

ALMEIDA, A. B.; BERTAN, C. M.; ROSSA, L. A.. F.; GASPAR, P. S.; BINELLI, M. et al. Avaliação da reutilização de implantes auriculares contendo norgestomet associados ao valerato ou ao benzoato de estradiol em vacas nelore inseminadas em tempo fixo. Brazilian Journal of Veterinary Research and Animal Science, v. 43, n. 4, p. 456-465, 2006. Disponível em: <http://dx.doi.org/10.11606/issn.16784456.bjvras.2006.26460> DOI: 10.11606/issn.1678-4456.bjvras.2006.26460

BARBOSA, C. F.; JACOMINI, J. O.; DINIZ, E. G.; SANTOS, R. M.; TAVARES, M. Inseminação artificial em tempo fixo e diagnóstico precoce de gestação em vacas leiteiras mestiças. Revista Brasileira de Zootecnia, v. 40, n. 1, p. 79-84, 2011. Disponível em: www.scielo.br/pdf/rbz/v40n1/v40n1a11.pdf

BÓ, G. A.; BARUSELLI, P. S. Synchronization of ovulation and fixed-time artificial insemination in beef cattle. Animal, v. 8, n. 1, p. 144-150, 2014.

Disponível em:

<https://doi.org/10.1017/S1751731114000822>

DOI:10.1017/S1751731114000822

BÓ, G. A.; BARUSELLI, P. S.; MAPLETOFT, R. J. Synchronization techniques to increase the utilization of artificial insemination in Beef and dairy cattle. Animal Reproduction, v. 10, n. 3, p. 137-142, 2013. Disponível em: $<$ http://www.cbra.org.br/pages/publicacoes/animalreproduction/issues/download/v10 n3/p137-142\%20(AR625).pdf>

CABRAL, J. F.; LEÃO, K. M.; SILVA, M. A. P.; BRASIL, R. B. Indução do estro em novilhas Nelore com implante intravaginal de progesterona de quarto uso. Revista Brasileira de Ciência Veterinária, v. 20, n. 1, p. 49-53, 2013. Disponível em: <http://dx.doi.org/10.4322/rbcv.2014.039> DOI: 10.4322/rbcv.2014.039

COLAZO, M. G.; MAPLETOFT, R. J. A review of current timed-AI (TAI) programs for beef and dairy cattle. The Canadian Veterinary Journal, v. 55, n. 8, p. 772-780, 2014. Disponível em: <https://www.ncbi.nlm.nih.gov/pmc/articles/PMC4095965/>

CORREA-OROZCO, A.; URIBE-VELÁSQUEZ, L.; PULGARÍN-VELÁSQUEZ, E. Factores que afectan la preñez en vacas Brahman sometidas a inseminación artificial a tiempo fijo. Revista MVZ Córdoba, v.18, n.1, 2013. Disponível em: <http://revistas.unicordoba.edu.co/revistamvz/mvz-181/v18n1a10.pdf>

CUNHA, R. R.; FERNANDES, C. A. C.; GARCIA, J. A. D.; GIOSO, M. M. Inseminação artificial em tempo fixo em primíparas Nelore lactantes acíclicas. Arquivo Brasileiro de Medicina Veterinária e Zootecnia, v. 65, n. 4, p. 1041-1048, 2013. Disponível em: <http://dx.doi.org/10.1590/S0102-09352013000400015> DOI: 10.1590/S0102-09352013000400015

ERENO, R. L.; BARREIROS, T. R. R.; SENEDA, M. M.; BARUSELLI, P. S.; 
PEGORER, M. F.; et al. Taxa de prenhez de vacas Nelore lactantes tratadas com progesterona associada à remoção temporária de bezerros ou aplicação de gonadotrofina coriônica eqüina. Revista Brasileira de Zootecnia, p. 1288-1294, 2007. Disponível em: <http://dx.doi.org/10.1590/S1516-35982007000600010> DOI: 10.1590/S1516-35982007000600010

FARIAS, M. C.; SOUTO, P. F. M. P.; FILHO, M. L. S.; LIMA, E. B.; SILVA, A. C. P.; et al. Influência da presença de muco e reutilização de dispositivos de progesterona sobre a taxa de concepção em vacas Nelore. Scientia Plena, v. 11, n. 4, 2015. Disponível em: <https://www.scientiaplena.org.br/sp/article/viewFile/2487/1182>

FERREIRA, M. C. N.; MIRANDA, R.; FIGUEIREDO, M. A.; COSTA, O. M.; PALHANO, $\mathrm{H}$. B. Impact of body condition on pregnancy rate of cows nellore under pasture in fixed time artificial insemination (tai) program. Semina: Ciências Agrárias, Londrina, v. 34, n. 4, p. 1861-1868, 2013. Disponível em: <http://dx.doi.org/10.5433/1679-0359.2013v34n4p1861> DOI: 10.5433/1679$0359.2013 \mathrm{v} 34 \mathrm{n} 4 \mathrm{p} 1861$

FREITAS, D. S.; CHALHOUB, M.; ALMEIDA, A. K. C.; SILVA, A. A. B.; SANTANA, R. C. M.; et al. Associação do diagnóstico precoce de prenhez a um protocolo de ressincronização do estro em vacas zebuínas. Revista Brasileira de Saúde e Produção Animal, v. 8, n. 3, 2007. Disponível em: <http://revistas.ufba.br/index.php/rbspa/article/view/721/461>

GOTTSCHALL, C. S.; ALMEIDA, M. R.; TOLOTTI, F.; MAGERO, J.; BITTENCOURT, H. B. et al. Avaliação do desempenho reprodutivo de vacas de corte lactantes submetidas à IATF a partir da aplicação do $\mathrm{GnRH}$, da manifestação estral, da reutilização de dispositivos intravaginais e da condição corporal. Acta Scientiae Veterinariae, v. 40, n. 1, 2012. Disponível em: http://www.ufrgs.br/actavet/40-1/PUB\%201012.pdf

GOTTSCHALL, C. S.; CANELLAS, L. C.; MARQUES, P. R.; ALMEIDA, M. R.; CRUZ, J. K. et al. Efeitos do número de dias pós-parto sobre o desempenho bioeconômico de vacas de corte submetidas à inseminação artificial em tempo fixo (IATF). Veterinária em Foco, v. 7, n. 1, p. 94-102, 2009. Disponível em: <revistas.bvs-vet.org.br/vetfoco/article/download/28354/29857>

GUNN, P. J.; LEMENAGER, R.; BRIDGES, A. Efficacy of the 5 Day CO-Synch Estrous Synchronization Protocol with or without the Inclusion of a Controlled Internal Drug Release Device in Beef Cows. Animal Industry Report, 2015. Disponível em: $<$ https://lib.dr.iastate.edu/cgi/viewcontent.cgi?referer=https://www.google.com.br/\&htt psredir $=1 \&$ article $=2057 \&$ context=ans_air $>$

MEDALHA A. G.; SOUZA, M. I. L.; SOUZA, A. S.; QUEIROZ, V. L. D.; FILHO, L. C. C. C. Reutilização de dispositivos intravaginais de progesterona, diâmetro folicular e comportamento estral na prenhes de vacas zebuínas. Enciclopédia biosfera, Centro Científico Conhecer, Goiânia, v. 10, n. 18, p. 2723- 2734, 2014. Disponível em:

<https://www.researchgate.net/publication/275519774_Reutilizacao_de_dispositivos 
intravaginais_de_progesterona_diametro_folicular_e_comportamento_estral_na_pr enhez_de_vacas_zebuinas>

MEDALHA, A. G.; SOUZA, M. I. L.; SOUZA, A. S.; SÁ FILHO, O. G.; QUEIROZ, V. L. D.; et al. Utilização do dispositivo intravaginal de progesterona, em até três usos, para inseminação artificial em tempo fixo de fêmeas Bos indicus. Revista brasileira de saúde e produção animal, v. 16, n. 2, 2015. Disponível em: <http://dx.doi.org/10.1590/S1519-99402015000200020> DOI: 10.1590/S151999402015000200020

MENEGHETTI, M.; VASCONCELOS, J. L. M. Mês de parição, condição corporal e resposta ao protocolo de inseminação artificial em tempo fixo em vacas de corte primíparas. Arquivo Brasileiro de Medicina Veterinária e Zootecnia, v. 60, n. 4, p. 786-793, 2008. Disponível em: <http://dx.doi.org/10.1590/S010209352008000400002> DOI: 10.1590/S0102-09352008000400002

PINTO-NETO, A.; SILVA, R. Z.; MOTA, M. F.; ALBERTON, J. Reutilização de implante intravaginal de progesterona para sincronização de estro em bovinos. Arq. Ciênc. Vet. Zool. UNIPAR, Umuarama, v. 12, n. 2, p. 169-174, 2009. Disponível em: <http://revistas.unipar.br/index.php/veterinaria/article/view/2972>

RODRIGUES, A. S.; OlIVEIRA, S. N.; LOIOLA, M. V. G.; FERRAZ, P. A.; CHALHOUB, M.et al. Contagem de folículos antrais em fêmeas Nelore submetidas a inseminação artificial em tempo fixo. Ciência Rural, v. 45, n. 4, p. 711, 2015. Disponível em: <http://dx.doi-org.ez372.periodicos.capes.gov.br/10.1590/01038478cr20140666> DOI: 10.1590/0103-8478cr20140666

SILVA, C. B.; ROSA, C. O.; SANTOS, M. M.; PELAQUIM, P. R.; PEDROSO, F. S.; et al. Efeito da reutilização do implante de progesterona multidose na taxa de concepção de fêmeas Bos indicus paridas submetidas à inseminação artificial em tempo fixo. Revista Acadêmica de Ciência Animal, v. 15, n. 2, p. 415-416, 2017. Disponível em: <https://periodicos.pucpr.br/index.php/cienciaanimal/article/view/17577>

SILVA, M. A. M.; PIMENTEL, L. A.; Mejoramiento genético en bovinos a través de la inseminación artificial y la inseminación artificial a tiempo fijo. Revista de Investigación Agraria y Ambiental, v. 8, n. 2, 2017. Disponível em: <http://hemeroteca.unad.edu.co/index.php/riaa/article/view/2050/2261 >

TORRES-JÚNIOR, J. R. S.; RIBEIRO, D. L. S.; PEREIRA, H. G.; FRANÇA, I. G. Mitos e verdades em protocolos de IATF. Revista Brasileira de Reprodução Animal, Belo Horizonte, v. 40, n. 4, p. 129-141, 2016. Disponível em: <http://www.cbra.org.br/portal/downloads/publicacoes/rbra/v40/n4/p129141\%20(RB691).pdf>

ZANGIROLAMO, A. F.; SILVA, N. C.; MOROTTI, C. S.; SENEDA, M. M. The Impact of Sanity on the Fertility of Cows Submitted to Reproductive Biotechnics. Revista Acadêmica de Ciência Animal, v. 15, n. 2, p. 91-98, 2017. Disponível em:<https://doi.org/10.7213/academica.15.S02.2017.A09>

DOI:

10.7213/academica.15.S02.2017.A09 